\title{
Big Data Analysis and Genetic Liability to Neuropsychiatric Disease
}

\author{
Panagiotis Roussos
}

The majority of risk genetic variants for common and complex neuropsychiatric traits lie within noncoding regions. Previous efforts have linked risk variants to specific genes by leveraging transcriptome data and expression quantitative trait loci. Most recently, the generation of large-scale epigenome data and the availability of epigenome quantitative trait loci provide a powerful discovery tool for assigning a functional role to the genetic variation in neuropsychiatric traits. In this talk, we will focus on advances in integration of epigenome datasets with the risk of common and complex neuropsychiatric traits.

P. Roussos $(\square)$

Department of Psychiatry, Friedman Brain Institute, Department of Genetics and Genomic Science and Institute for Multiscale Biology; Icahn School of Medicine at Mount Sinai, New York, NY, USA

e-mail: panagiotis.roussos@mssm.edu 